\title{
INFECCIÓN DE FRACTURAS TIBIALES ABIERTAS CON Y SIN FIJACIÓN EXTERNA PROVISIONAL HOSPITAL DE SAN JOSÉ 2008-2009
}

Julio César Ramírez Lamas MD*, Juan Karlos Torres Higuita MD*, Juan Manuel Nossa MD**, Carlos Mario Olarte MD***, Guillermo Rueda MD****

\section{Resumen}

La infección relacionada con la fractura abierta de tibia constituye una complicación importante ya que tiene relación directa con el pronóstico y el resultado funcional. En nuestro hospital se desconoce su incidencia y si ocurre con mayor frecuencia con el uso de fijador externo. Se realizó estudio observacional tipo cohorte prospectiva en pacientes mayores de 16 años que ingresaron al servicio de ortopedia y traumatología del Hospital de San José con diagnóstico de fractura abierta de tibia en el período abril 2008 a abril 2009. Hubo seguimiento desde su ingreso hospitalario hasta los controles posteriores. Se evaluaron un total de 71 casos en los cuales predominaron el sexo masculino $(84,5 \%)$ y el accidente de tránsito como mecanismo de trauma $(\mathbf{7 8 , 8} \%)$. La incidencia total de infección en la población a estudio fue de $9,8 \%$ y el germen aislado con mayor frecuencia fue Staphylococcus aureus en $(57,1 \%)$. Todos los infectados fueron manejados con fijación externa provisional y en su mayoría fueron grado IIIA en la clasificación de Gustilo $(36,6 \%)$, siendo las fracturas $I I$ y $I I I$ en las que se presentó con mayor frecuencia. Concluimos que todos los casos de infección se documentaron con fijación externa y debe ser considerada en el manejo de estos pacientes.

Palabras clave: fracturas de la tibia, fijación de fractura, infección de heridas.

Abreviatura: FAT, fractura abierta de tibia.

\section{INFECTION OF TIBIA OPEN FRACTURES WITH AND WITHOUT PROVISIONAL EXTERNAL FIXATION HOSPITAL DE SAN JOSÉ 2008-2009}

\section{Abstract}

Infection associated to open tibia fractures is considered an important complication for it is directly related to prognosis and functional results. Its incidence is unknown in our hospital and is mainly associated with the use of an external fixator. An observational prospective cohort study was conducted in patients older than 16 years of age admitted to the orthopedics and traumatology service at the Hospital de San José with a diagnosis of open tibia fracture, between April 2008 and April 2009. Follow-up was carried out from admission to control visits. A total number of 71 cases were assessed with male predominance $(84,5 \%)$ and car accident as trauma mechanism $(\mathbf{7 8 , 8 \%})$. Total incidence of infection in the study population was $\mathbf{9 , 8 \%}$ and the most frequently identified organism was Staphillococcus aureus in $\mathbf{5 7 , 1 \%}$. All affected patients had been managed with a provisional external fixation and most of them were categorized grade IIIA on the Gustillo classification (36,6\%). The majority were grade $I I$ and $I I I$ fractures. We conclude that all cases of infection were associated to external fixation, thus, it must be considered in the management of these patients.

Key words: tibia fractures, fracture fixation, wound infection.

Fecha recibido: diciembre 18 de 2009 - Fecha aceptado: enero 25 de 2010

* Residente IV de Ortopedia, Fundación Universitaria de Ciencias de la Salud. Hospital de San José, Bogotá DC. Colombia.
** Instructor de cadera y trauma. Hospital de San José, Bogotá DC. Colombia.

*** Instructor de trauma. Hospital de San José, Bogotá D.C. Colombia. ***** Instructor Especialista de cadera, Hospital de San José, Bogotá DC. Colombia. 


\section{Introducción}

Las fracturas abiertas se definen como aquellas que se comunican con el medio ambiente, resultando tanto en contaminación con microorganismos así como compromiso de la vascularización que suple la región afectada; por tanto, están asociadas con un riesgo mayor de infección y a medida que ésta aumenta se incrementan las fallas en la consolidación. Además, el cartílago, los tendones y nervios pueden ser expuestos a lesión, lo que tiene consecuencias para el pronóstico funcional del paciente. ${ }^{1}$

Las FAT constituyen un problema frecuente en nuestro medio y ocurren como consecuencia de traumas de alta energía, por lo regular ocasionados por accidentes de tránsito. ${ }^{2}$ Se caracterizan por lesión de tejidos blandos de grado variable debido a su localización subcutánea. ${ }^{3,4}$ Todas las FAT se consideran heridas contaminadas y requieren lavados quirúrgicos previos a la fijación provisional o definitiva. El porcentaje de infección resultante varía de acuerdo con el grado de compromiso de los tejidos blandos, para lo cual se utiliza la clasificación de Gustilo. ${ }^{3}$ Se ha reportado que el riesgo de infección varía de 0 a $2 \%$ para las tipo $I, 2$ a $10 \%$ para el $I I$ y hasta 10 a $50 \%$ para el tipo III. Dentro de las técnicas de reducción para manejo de la fractura se incluye la fijación interna con clavos, placas o tornillos y la fijación externa. Esta última puede ser útil en las FAT cuando hay lesión severa de los tejidos blandos y contaminación, ya que no compromete la vascularización ósea; además, técnicamente no es demandante y se asocia con mínima pérdida de sangre, así como puede ser aplicada distal a la lesión sin interferir con el manejo de la herida.,

Los fijadores externos tienen dos indicaciones: ${ }^{7}$ para el control del daño ortopédico como dispositivo temporal y para el manejo definitivo de fracturas y reconstrucción postraumática. Entre las complicaciones del uso de la fijación externa se han documentado la infección del tracto de los clavos y el mal alineamiento de la fractura. Estas se pueden evitar con la selección adecuada del paciente y la implementación de un protocolo para la colocación del fijador externo. Se ha reportado infección relacionada con FAT hasta de $65 \% .{ }^{8,9,10}$ Es promovida por la contaminación bacteriana, la colonización de la herida, la presencia de espacio muerto con tejido desvitalizado, material extraño y el compromiso de la respuesta del huésped resultante de la pobre vascularización. Para prevenir la infección clí- nica se deben administrar antibióticos, realizar un adecuado desbridamiento de la herida, cobertura con tejidos blandos y estabilización de la fractura. En el manejo de una FAT los antibióticos no deben ser profilácticos y más bien su uso debe ser considerado terapéutico. Se deduce que la alta frecuencia de este tipo de evento debe considerarse como indicador de importancia en cualquier institución que maneje pacientes con este tipo de características.

El reconocimiento precoz de la infección se basa en los signos y síntomas de la inflamación que se detectan con facilidad; sin embargo, es necesaria una anamnesis exhaustiva. La utilidad de los cultivos iníciales obtenidos al ingreso del paciente, durante el procedimiento quirúrgico, antes y después del desbridamiento de la fractura, es controvertida ya que a menudo fallan en indicar el organismo causal. ${ }^{11,12}$ En el estudio de Patzakis (2000) sólo 3 (18\%) de 17 infecciones que se desarrollaron en una serie de 171 fracturas abiertas fueron causadas por el organismo identificado en el cultivo inicial. ${ }^{13}$ Se ha mostrado que la mayoría son originadas por bacterias de carácter nosocomial. ${ }^{14}$ En un estudio llevado a cabo por Carsenti-Etesse y colaboradores, 92\% de las 38 fracturas abiertas se infectaron mientras el paciente se encontraba hospitalizado. ${ }^{15}$

Referente al manejo antibiótico, se ha demostrado una marcada reducción en el porcentaje de infección con el uso de cefalotina [2,4\% (2/84)] comparada con los que no reciben antibióticos $[13,9 \%(11 / 79)]$ o bien penicilina y estreptomicina $(9,8 \%)^{9}$ y deben administrarse antes del desbridamiento de la herida. En el servicio de ortopedia del Hospital de San José existe un protocolo de manejo de las fracturas abiertas de acuerdo con la clasificación de Gustilo $^{16}$ : a) grado I: cefalosporinas de primera generación; b) grado II: cefalosporinas de primera generación más aminoglucósidos y c) grado III: cefalosporinas asociadas con aminoglucósidos para mejorar el cubrimiento de gram negativos y en sospecha de contaminación por anaerobios se debe agregar penicilina cristalina. A todos los pacientes se les hace profilaxis antitetánica de acuerdo con el esquema de vacunación previa. Cuando se inician los antibióticos después de tres horas de ocurrida la lesión, aumenta el riesgo de infección. ${ }^{10}$

En el Hospital de San José se reciben numerosos pacientes con este diagnóstico, causados en su gran mayoría por 
traumas de alta energía (accidentes de tránsito). Es relevante la evaluación de la incidencia de infección en estos casos, pues se relaciona con el pronóstico y el resultado final. Así mismo, puede ser un indicador del éxito del tratamiento instaurado. Por tal motivo, el interés y el objetivo del presente estudio se basa en la necesidad de establecer la incidencia de infección de las FAT de acuerdo con la clasificación de Gustilo, tanto en quienes se realiza fijación externa provisional y después osteosíntesis definitiva, como en los pacientes a los que se practica osteosíntesis temprana definitiva, con el fin de aportar información acerca de la frecuencia de este tipo de complicaciones y caracterizar la población que la padece.

\section{Métodos}

Se realizó un estudio observacional tipo cohorte prospectiva de pacientes mayores de 16 años que ingresaron al Hospital de San José en el período abril 2008 a abril 2009, con diagnóstico de FAT y que fueron manejados en el servicio de ortopedia y traumatología del hospital. Los investigadores hicieron seguimiento desde el ingreso hospitalario hasta los controles posteriores. Los criterios de inclusión fueron pacientes mayores de 16 años, FAT sin criterio de amputación, seguimiento posoperatorio de seis meses y estudios imagenológicos completos. Se recolectó información sociodemográfica (sexo y edad), relacionada con la fractura (clasificación de Gustilo, extremidad fracturada), necesidad del uso de fijación externa provisional, mecanismo del trauma y relación con la presencia de infección (germen aislado). Se consideró que un paciente presentaba infección con hallazgos clínicos como: calor, rubor, secreción purulenta o presencia de síntomas sistémicos de infección, así como el aislamiento positivo reportado por el laboratorio de la institución. Para el análisis de la información se usó el paquete estadístico STATA 10. El protocolo tuvo la aprobación del comité de investigaciones y ética de la Facultad de Medicina de la Fundación Universitaria de Ciencias de la Salud y fue clasificada como una investigación sin riesgo.

\section{Resultados}

Se evaluaron un total de 71 pacientes con diagnóstico de FAT manejados con y sin fijación externa provisional en el Hospital de San José durante el período de estudio. En cuanto al género, el porcentaje de hombres fue $84,5 \%$ y la media de edad 38.9 años (DE 15.1 años; rango 16-78). Con respecto al mecanismo de trauma, el de mayor frecuencia fue el causado por accidentes de tránsito en un $78,8 \%$; otros incluían traumas directos, caídas y heridas por arma de fuego. El promedio de lavados quirúrgicos previos a la fijación definitiva fue 1.8 (DE 1.19; rango 1-7 lavados). El tiempo promedio transcurrido para el manejo quirúrgico definitivo fue de cuatro días (DE 4.56 días; rango 0.25-27 días) y 32,3\% de los pacientes presentaron politraumatismo. Con respecto a la localización de la fractura, fue más frecuente en la diáfisis de la tibia en 57,7\% del total de pacientes del estudio y en $71 \%$ en quienes se usó fijador externo provisional. Las clasificadas como Gustilo IIIA fueron las más frecuentes en la población atendida $(36,6 \%)$. En cuanto al método quirúrgico definitivo, en el 56\% se usó enclavijamiento endomedular, seguido por uso de placas en un 27\%. La osteosíntesis definitiva mediante este último procedimiento se realizó sólo en FAT Gustilo I, II y IIIA. La información referente al manejo quirúrgico definitivo puede verse en la Figura 1.

A 46,4\% se le realizó fijación externa provisional (33 casos) y en ellos se encontró que fueron mayores la frecuencia de Gustilo II y III, el número promedio de lavados quirúrgicos, el tiempo del manejo quirúrgico definitivo, la mayor frecuencia de complicaciones no infecciosas y el politraumatismo. El promedio de tiempo para la realización del manejo quirúrgico definitivo en los pacientes con fijación externa provisional fue de 7.28 días (DE 4.4 días; rango 4 a 15 días). De los que no recibieron fijación externa provisional el promedio de manejo quirúrgico definitivo fue de 1.58 días (DE 1.25 días; rango 0.25 a 5 días). La información relacionada con estas variables en pacientes con y sin tutor, puede verse en la Tabla 1.

La incidencia total de infección en la población estudiada fue de $9,85 \%$ y se presentó en los primeros 30 días. Se aisló como germen causal al Staphylococcus aureus en el 57,14\%. Todos los infectados recibieron manejo con fijación externa provisional ( $21,2 \%$ de 33 pacientes). La mediana de edad fue 34 años (rango 26-56 años). En cuanto a la incidencia de infección por gravedad de la lesión, los grados II y III presentaron este tipo de complicación, en comparación con los grado I donde no hubo casos. La información sobre la clasificación general de las fracturas y la infección se encuentra en la Figura 2. 


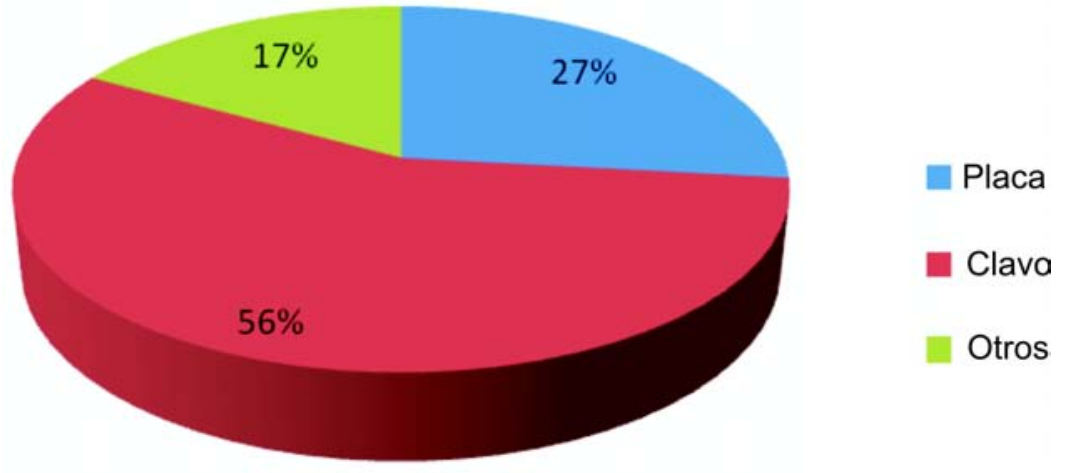

Figura I. Manejo quirúrgico definitivo en FAT.

Tabla I. Características sociodemográficas y clínicas según manejo quirúrgico con y sin fijación externa

\begin{tabular}{|c|c|c|c|c|}
\hline Fijación externa & Sí $(n=33)$ & & No $(n=38)$ & \\
\hline Edad en años (media-DE) & 37.5 & 14.4 & 40 & 15.8 \\
\hline \multicolumn{5}{|l|}{ Género $(n-\%)$} \\
\hline Masculino & 27 & 81.8 & 33 & 86.8 \\
\hline Femenino & 6 & 18.1 & 5 & 13.1 \\
\hline \multicolumn{5}{|l|}{ Mecanismo de trauma (n-\%) } \\
\hline Accidente de trabajo & 0 & 0 & 2 & 5.2 \\
\hline Accidente de tránsito & 27 & 81.8 & 29 & 76.3 \\
\hline Caídas & 3 & 9.1 & 3 & 7.8 \\
\hline Herida por proyectil/arma de fuego & 1 & 3.0 & 1 & 2.6 \\
\hline Trauma & 2 & 6.1 & 3 & 7.8 \\
\hline \multicolumn{5}{|l|}{ Clasificación de Gustilo (n-\%) } \\
\hline Grado I & 7 & 21.2 & 16 & 42.1 \\
\hline Grado II & 5 & 15.1 & 8 & 21.1 \\
\hline Grado IIIA & 13 & 39.3 & 13 & 34.2 \\
\hline Grado IIIB & 5 & I5.I & 1 & 2.6 \\
\hline Grado IIIC & 3 & 9.1 & 0 & 0 \\
\hline \multicolumn{5}{|l|}{ Localización de la fractura (n-\%) } \\
\hline Proximal & 5 & 15.1 & 5 & 13.1 \\
\hline Diafisiaria & 14 & 42.4 & 27 & 71.05 \\
\hline Distal & 11 & 33.3 & 6 & 15.7 \\
\hline Sin dato & 3 & 9.09 & 0 & 0 \\
\hline Número de lavados quirúrgicos (media-DE) & 2.6 & 1.2 & 1.1 & 0.4 \\
\hline \multicolumn{5}{|l|}{ Clasificación de Tscherne (n-\%) } \\
\hline Tscherne I & 12 & 36.3 & 34 & 91.8 \\
\hline Tscherne II & 19 & 57.5 & 3 & 8.1 \\
\hline Tscherne III & 2 & 6.1 & 0 & 0 \\
\hline Tscherne IV & 0 & 0 & 0 & 0 \\
\hline \multicolumn{5}{|l|}{ Días hasta el manejo quirúrgico definitivo } \\
\hline (media- DE) & 6.3 & 5.3 & 1.5 & 1.2 \\
\hline Presencia de infección (n-\%) & 7 & 21.2 & 0 & 0 \\
\hline Complicaciones no infecciosas (n-\%) & 12 & 36.3 & 4 & 10.5 \\
\hline Politraumatismo (n-\%) & 14 & 43.7 & 8 & 22.2 \\
\hline
\end{tabular}


Del total de pacientes infectados, a tres (grados II, IIIA y IIIB) se les realizó lavado quirúrgico provisional en urgencias como manejo inicial y se pasó a tratamiento quirúrgico provisional con fijación externa en un promedio de ocho horas. El número de lavados quirúrgicos realizados fue mayor para el grupo manejado con fijación externa provisional, en concordancia con la gravedad de la fractura. El tiempo promedio en días del tratamiento quirúrgico definitivo con fijación fue de 6.3 y sin fijación 1.5; con infección fue de 7.2 y sin infección 3.68. Las complicaciones no infecciosas durante los controles, como pseudoartrosis y defecto de cobertura, entre otras, se encontraron en mayor porcentaje en quienes se usó fijación externa $(36,6 \%)$.

\section{Discusión}

Los resultados encontrados en el presente estudio muestran una mayor frecuencia de FAT en pacientes de sexo masculino y entre la tercera y cuarta década de la vida, en su mayoría debidas a accidentes de tránsito. La localización anatómica más frecuente fue la diáfisis de la tibia y guarda estrecha relación con el mecanismo de trauma, similar con lo reportado en la literatura. ${ }^{2,17} \mathrm{La}$ incidencia de infección fue de $9,8 \%$ y el uso de fijación externa se asoció con infección en el $21,2 \%{ }^{1}$ siendo todas las fracturas abiertas grados $I I(14,2 \%)$ y $I I I(87,5 \%)$.

En cuanto a los cultivos, el microorganismo aislado en $57,1 \%$ fue Staphylococcus aureus como es referido en la literatura, ${ }^{8,18}$ en cultivos realizados cuando la infección se diagnostica clínicamente. Es importante señalar que la utilidad de los cultivos iniciales obtenidos al ingreso, durante el procedimiento quirúrgico o antes y después del desbridamiento de la fractura, es controvertida ya que con frecuencia falla en indicar el organismo causal. ${ }^{11,12}$ En un estudio aleatorio prospectivo y doble ciego realizado por Patzakis y colaboradores en el $2000^{13}$, sólo el $18 \%$ de las infecciones en fracturas abiertas eran causadas por el organismo identificado en el cultivo inicial; a esto hay que añadir el uso inicial de antibióticos de amplio espectro, los múltiples desbridamientos y los patógenos nosocomiales como factores de error para determinar el momento adecuado para la toma de un cultivo que sea útil para aplicación clínica.

Como manejo quirúrgico definitivo se utilizó enclavijamiento endomedular (la mayoría de veces rimado) en el 56\% de FAT tipos Iy IIIA, y presentaron infección en un 23,5\% luego de la conversión. Sin embargo, la experiencia y la incidencia de infección con el enclavijamiento endomedular rimado es limitada en la literatura mundial y este dato no es comparable. Un metanálisis del manejo de las FAT demostró que los clavos endomedulares no rimados reducen el riesgo de cirugía de revisión, mala-unión e infección superficial al compararla con la fijación externa. ${ }^{19}$ Una considerable proporción de las complicaciones debidas a la fijación externa se pueden asociar con la transición a otra forma de fijación. La infección se ha reportado en un 50\% luego de la conversión de fijación externa a enclavijamiento endomedular tardío. ${ }^{11,20}$ En nuestro estudio esto se observó en el 21,2\%. Sin embargo, en estas series la infección se asociaba con infección primaria en los trayectos de los clavos, lo cual no fue analizado en el presente estudio.

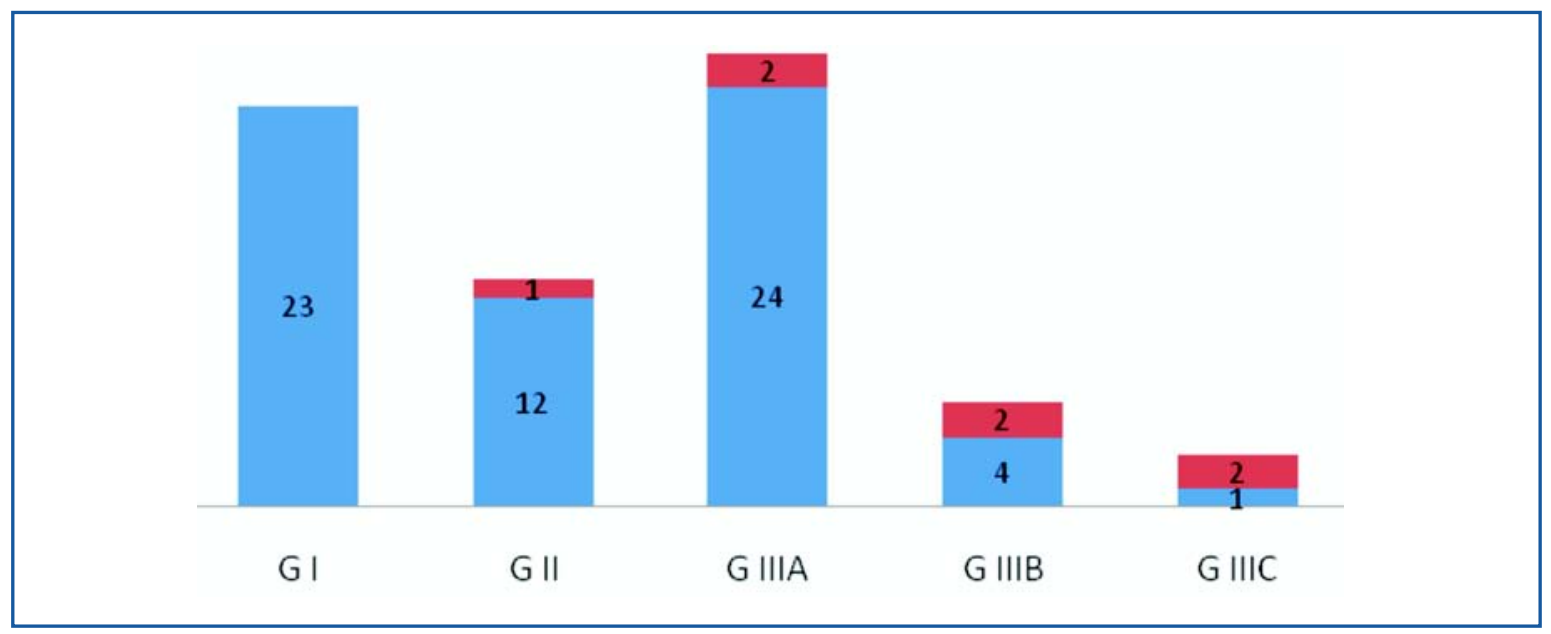

Figura 2. Casos de infección según la clasificación de Gustilo. 
El riesgo de infección clínica depende de la severidad de la lesión y el grado de compromiso de las partes blandas. Bowen y Widmaier ${ }^{21}$ estudiaron 174 pacientes con fracturas abiertas de huesos largos y encontraron que no sólo la clasificación de Gustilo y Anderson, sino también el número y compromiso por las comorbilidades (factores de riesgo) estuvieron asociados con la infección. Bhandari y colaboradores revisaron la literatura en un intento de definir mejor el riesgo de infección y la falta de unión con fijación externa temporal antes del clavo endomedular en fracturas diafisiarias de fémur y tibia. ${ }^{22}$ Buscaron determinar la relación entre el tiempo en que el fijador es colocado y la tasa de infección. En nueve estudios encontraron el 3,7\% de infección en 191 fracturas con menos de 28 días de fijación externa; esta frecuencia subía a 22,1\% cuando era mayor de 28 días. Algunos pacientes con conversiones tardías tenían múltiples traumas o problemas médicos que no permitían la conversión temprana, lo que incrementa la tasa de infección. En nuestro estudio no se puede realizar esta comparación porque el promedio de tiempo para la realización del manejo quirúrgico definitivo en los pacientes con fijación externa provisional fue de 7.28 días. La pseudoartrosis y la infección son las complicaciones más frecuentes en estos pacientes y deben correlacionarse no sólo con la condición de los tejidos blandos debido al trauma inicial (pérdida ósea, falta de cubrimiento cutáneo, contaminación) sino a la condición médica del paciente (comorbilidades) que puede tener repercusión en la evolución clínica y en el pronóstico, además del manejo quirúrgico que se realice ya sea fijación externa provisional o manejo quirúrgico definitivo con osteosíntesis y el momento en que se efectúa. Entre las limitaciones del presente trabajo fue la falta de identificación de las comorbilidades y la demora del tratamiento quirúrgico definitivo. Además algunos pacientes fueron remitidos a otras instituciones y no se pudo determinar si hubo infección, lo que contribuyó a disminuir el número de casos. En conclusión, todas las infecciones se presentaron en pacientes con fijación externa provisional.

\section{Referencias}

1. Charalampos G. Zalavras, and Michael J. Patzakis. Open fractures: evaluation and management. Journal American Academic Orthopedic Surgery. $2003 ; 11: 212-9$
2. Blanco-Blanco JF, Galea RR, Martin PH, Ratero DB, Moro JA. Tratamiento de las fracturas abiertas de la tibia mediante enclavado endomedular encerrojado no fresado. Informe de 20 casos. Acta Ortop Mex. 2003; $17(2): 81-4$

3. Charalampos G. Zalavras, Randall E. Marcus, L. Scott Levin, and Michael J. Patzakis. Management of open fractures and subsequent complications. The Journal of Bone \& Joint Surgery. 2007;89(4):884-95.

4. Blick SS, Brumback RJ, Poka A, Burgess AR, Ebraheim NA. Compartment syndrome in open tibial fractures. J Bone Joint Surg Am. 1986;68:1348-53.

5. Edwards CC, Simmons SC, Browner BD, Weigel MC. Severe open tibial fractures: Results treating 202 injuries with external fixation. Clin Orthop. 1988;230:98-115.

6. Marsh JL, Nepola JV, Wuest TK, Osteen D, Cox K, Oppenheim W. Unilateral external fixation until healing with the dynamic axial fixator for severe open tibial fractures. J Orthop Trauma. 1991;5:341-8.

7. Bruce H. Ziran, Wade R. Smith, Jeff O. Angle, and Paul Tornetta. III: External Fixation: How to Make It Work An Instructional Course Lecture, J Bone Joint Surg Am. 2007;89:1620-32

8. Gustilo RB, Anderson JT. Prevention of infection in the treatment of one thousand and twenty-five open fractures of long bones: Retrospective and prospective analyses. J Bone Joint Surg Am. 1976;58:453-8

9. Patzakis MJ, Harvey JP Jr, Ivler D. The role of antibiotics in the management of open fractures. J Bone \& Joint Surg Am. 1974;56:532-41

10. Patzakis MJ, Wilkins J. Factors influencing infection rate in open fracture wounds. Clin Orthop. 1989; 243: 36-40

11. Fischer MD, Gustilo RB, Varecka TF. The timing of flap coverage, bonegrafting, and intramedullary nailing in patients who have a fracture of the tibial shaft with extensive soft-tissue injury. J Bone Joint Surg Am. 1991;73:1316-22.

12. Lee J. Efficacy of cultures in the management of open fractures. Clin Orthop. 1997;339:71-5

13. Patzakis MJ, Bains RS, Lee J, et al. Prospective, randomized, double-blind study comparing single-agent antibiotic therapy, ciprofloxacin, to combination antibiotic therapy inopen fracture wounds. J Orthop Trauma 2000;14:529-33.

14. Álvarez LópezA, Casanova Morote C, García Lorenzo Y. Fracturas diafisiarias abiertas de tibia. Rev Cubana Ortop Traumatol 2004;18(1)24-8.

15. Carsenti-Etesse H, Doyon F, Desplaces N, Gagey O, Tancrede C, Pradier C, Dunais B, Dellamonica P. Epidemiology of bacterial infection during management of open leg fractures. Eur J Clin Microbiol Infect Dis. 1999; 18:315-23.

16. Olarte MD, Fracturas abiertas, Guía de Manejo Servicio de Ortopedia y Traumatología Hospital de San José. Circulación interna. 2003.

17. Rockwood X, Green X. Fractures in adults. $5^{\mathrm{a}}$ ed. Madrid: Elsevier. 2008.

18. Gustilo RB, Mendoza RM, Williams DN. Problems in the management of type III (severe) open fractures: a new classification of type III open fractures. J Trauma. 1984;24:742-6

19. Bhandari M, Guyatt GH, Swiontkowski MF, Schemitsch EH. Treatment of open fractures of the shaft of the tibia. J Bone Joint Surg Br. 2001;83:62-8.

20. McGraw JM, Lim EV. Treatment of open tibial-shaft fractures: External fixation and secondary intramedullary nailing. J Bone Joint Surg Am. 1988;70:900-11.

21. Bowen TR, Widmaier JC. Host classification predicts infection after open fracture. Clin Orthop Relat Res. 2005;433:205-11

22. Bhandari M, Zlowodzki M,Tornetta P III, Schmidt A, Templeman DC Intramedullary nailing following external fixation in femoral and tibial shaft fractures. J Orthop Trauma. 2005;19:140-4. 